\title{
Computed bound and continuum electronic states of the nitrogen molecule
}

\author{
Jonathan Tennyson a and Duncan A. Little \\ Department of Physics and Astronomy, University College London, London WC1E 6BT, UK
}

\begin{abstract}
The dissociative recombination (DR) of $\mathrm{N}_{2}^{+}$is important for processes occurring in our atmosphere. However, it is not particularly well characterised, experimentally for the vibrational ground state and, theoretically for the $v \geq 4$. We use the R-matrix method to compute potential energy curves for both the bound Rydberg states of nitrogen and for quasi-bound states lying in the continuum. Use of a fine mesh of internuclear separations allows the details of avoided crossings to be determined. The prospects for using the curves as the input for DR calculations is discussed.
\end{abstract}

\section{Introduction}

Nitrogen $\left(\mathrm{N}_{2}\right)$ is the major constituent of the Earth's atmosphere and is present in significant quantities in the atmospheres of other planets. This means that dissociative recombination (DR) of $\mathrm{N}_{2}^{+}$is an important process when studying both naturally occurring processes in planetary ionospheres and for plasma flows related to planetary atmospheric entries [1]. Experimental studies of $\mathrm{N}_{2}^{+} \mathrm{DR}$ are hindered by the lack of a permanent dipole moment; the system does not radiatively cool. Collisional quenching has proven to be inefficient in cooling the system to its ground state [2]. As a result only limited and relatively uncertain data is available experimentally for this process [3, 4]. Furthermore, re-entry studies require DR data not just for the vibrational ground state but for a range of vibrationally excited state. This information is hard to extract experimentally even for species whose rotation-vibration population is easy to control. These issues place a particular emphasis on computational studies of $\mathrm{N}_{2}^{+} \mathrm{DR}$.

Guberman [5-8] has performed a series of theoretical studies on $\mathrm{N}_{2}^{+}$DR based on the use of multireference configuration interaction (MRCI) curves that he calculated using the quantum chemistry package MOLPRO [9]. MOLPRO is a highly optimised program which is excellent for treating molecular valence states but is not appropriate for studying highly diffuse Rydberg series.

We have therefore undertaken a series of studies of the bound and continuum states [10] of $\mathrm{N}_{2}$ based on the use of scattering calculations. These calculations gives comprehensive results for the Rydberg states while also treating the associated valence states. In this report we summarise progress on this work.

\footnotetext{
${ }^{a}$ Corresponding author: j.tennyson@ucl.ac.uk
}

This is an Open Access article distributed under the terms of the Creative Commons Attribution License 4.0, which permits unrestricted use, distribution, and reproduction in any medium, provided the original work is properly cited. 


\section{Method}

\subsection{General consideration}

Within the close-coupling expansion it is usual to express the wavefunction, $\Psi_{k}$, for an electron scattering from an $N$-electron target, in the form:

$$
\begin{aligned}
\Psi_{k}^{N+1}\left(x_{1}, \ldots, x_{N+1}\right) & =\mathcal{A} \sum_{i j} a_{i j k} \phi_{i}^{N}\left(x_{1}, \ldots, x_{N}\right) u_{i j}\left(x_{N+1}\right) \\
& +\sum_{i} b_{i k} \chi_{i}^{N+1}\left(x_{1}, \ldots, x_{N+1}\right)
\end{aligned}
$$

where the wavefunctions of target state $i$ is $\phi_{i}^{N}$, which is itself usually represented by a configuration interaction (CI) expansion. In this expression the $u_{i j}$ are continuum functions, for which we employ sets of Gaussian type orbitals (GTOs) placed on the centre-of-mass [11]. The antisymmetriser, $\mathcal{A}$, ensures that this wavefunction product satisfies the Pauli Principle, although this introduces further complications with the phase of the wavefunctions which need to be treated with care [12]. The second term in Eq. (1) involves placing the "scattering" electron in target orbitals which both relaxes the artificial orthogonalisation between the continuum and target orbitals, and allows for polarisation of the target in response to the extra electron.

Our calculations are performed using the R-matrix method, see reviews [13, 14], as implemented in the UKRMol codes [15] which contains a module, SCATCI [16], specifically constructed for efficient computation of wavefunctions in the form of Eq. (1), and which is particularly efficient for complicated target wavefunctions. Other algorithmic developments $[17,18]$ have further improved the computational performance of this key part of the code and, as will be seen below, has allowed the $\mathrm{N}_{2}$ problem to be systematically treated on a very dense grid of internuclear separations, $R$.

The R-matrix method divides space into an inner region, containing the $N$-electron target wavefunction, and an outer region. Although the method is designed for treating scattering problems it also appropriate for studying diffuse bound states $[19,20]$ by performing calculations at negative scattering energies [19]. Amongst other things this means that bound and continuum (resonance) states can be treated on the same footing.

In fact, the wavefunction of Eq. (1) is valid for the $(N+1)$-electron electronically bound state problem without the need for the division of space into regions. It is therefore possible to use the UKRMol codes in "quantum chemistry" mode were integration is performed over all space, instead of just inside the R-matrix sphere, and only states lying below the first ionisation threshold are considered.

Quasi-bound states, resonances, lying above the threshold to ionisation must however be treated as solutions of the scattering problem, see Ref. [21] for a discussion of this. In this case it is necessary to locate and characterise both the position and width (lifetime) of the resonances. The UKRMol codes contain three different modules for resonance characterisation: RESON [22] which performs a BreitWigner fit to the eigenphase sums [23], TIMEDEL [24] which fits to eigenvalues of the time-delay matrix [25, 26] and the QB method [27] which is a direct R-matrix specific procedure [28]. In practice we favour the use of the time-delay method [10] as it copes with the multiple, overlapping resonances seen in ionic targets better than Breit-Wigner fitting. The neglect of the long-range potentials in the outer region by the QB method leads to small but significant errors in the resonance positions.

\subsection{Specifics of the calculations}

Our target calculation was constructed using multi-configuration self-consistent field (MCSCF) molecular orbitals (MOs) generated by MOLPRO and a cc-pVQZ GTO basis. The MCSCF calculation froze the four $1 \mathrm{~s}$ electrons and considered a complete active space (CAS) with 9 electrons distributed 


\section{DR2013}

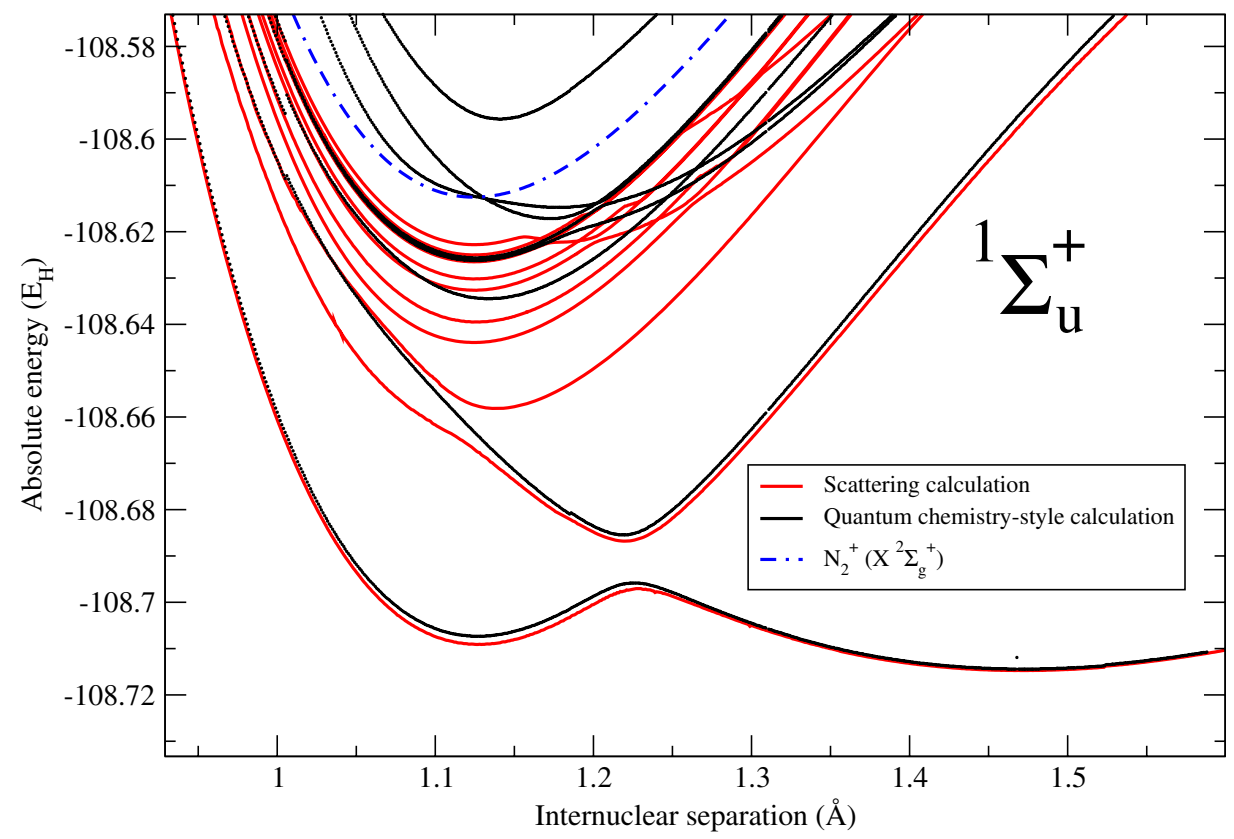

Figure 1. Potential energy curves of excited states of $\mathrm{N}_{2}$ with ${ }^{1} \Sigma_{\mathrm{u}}^{+}$symmetry. The dashed line denotes the ground state of $\mathrm{N}_{2}^{+}$. The other curves are obtained from a full R-matrix scattering calculation (red) and a inner region "quantum chemistry-style" calculation (black).

between next three $\sigma_{\mathrm{g}}$, three $\sigma_{\mathrm{u}}$, one $\pi_{\mathrm{u}}$ and one $\pi_{\mathrm{g}}$ orbitals. The R-matrix target wavefunction was built using the same CAS and was found to give potential energy curves for the first three electronic states of $\mathrm{N}_{2}^{+}, \mathrm{X}^{2} \Sigma_{\mathrm{g}}^{+}, \mathrm{A}^{2} \Pi_{\mathrm{u}}$ and $\mathrm{B}^{2} \Sigma_{\mathrm{u}}^{+}$, in excellent agreement with those generated by inverting experimental data, see Ref. [10] for further details. In practice, one hundred target states were actually considered in the close coupling expansion to allow for polarisation effects, although only those states target listed above are accurately represented within our model.

The scattering calculations used GTOs with s, p, d, f and g symmetry to represent the continuum orbitals meaning that states with symmetry up to $\Gamma_{\mathrm{g}}$ and with $\ell \leq 4$ were generated in the calculation. Calculations were performed at a fine grid of points in $0.001 \AA$ steps, with finer steps used to characterise key parts of curves of importance for the DR process. The main focus of cited work [10] has been on the region about equilibrium, $0.8 \AA \leq R \leq 1.999 \AA$. Calculations have also performed at larger $R$ to help characterise asymptotes which is important for the calculation of DR branching ratios. However, we note that the use of bond-centred functions to represent the scattering (Rydberg) electron becomes less efficient as the molecule is stretched and this can lead to some artefacts at large $R$ [29].

For further technical details see the paper reporting the results of the bound and continuum [10] state calculations.

\section{Results}

Figure 1 compares bound state curves of ${ }^{1} \Sigma_{u}^{+}$symmetry computed using a full scattering calculation (in red) and the same wavefunctions as part of a quantum chemistry-style calculation (in black). Although the curves appear continuous, the plots actually just give calculated points. It can be seen that the high resolution in $R$ allows structures in the curves, largely due to avoided crossings, to be well-characterised. 


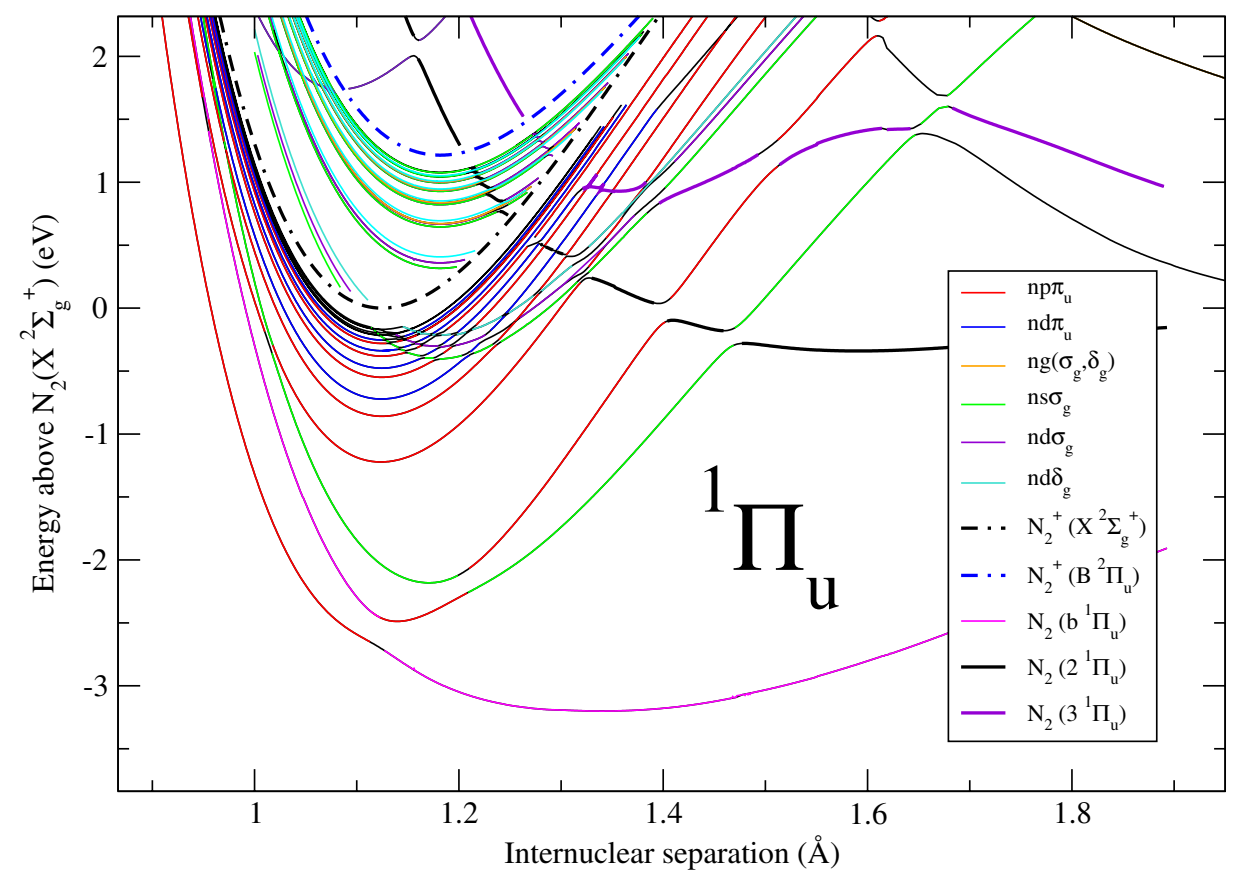

Figure 2. Bound and continuum states of $\mathrm{N}_{2}$ with ${ }^{1} \Pi_{\mathrm{u}}$ symmetry. The chain lines denote states of $\mathrm{N}_{2}^{+}$. Colours are used to give the (approximate) partial wave description of each Rydberg series.

Such couplings between different curves is one of the inputs that is required for a comprehensive treatment of DR for the system.

It is clear that the scattering calculations give many more states than the simpler quantum chemistrystyle treatment. This is because the numerical solution of the outer region problem performed in the scattering calculation yields, in principle, all states for the channels included in the calculation. In practice we have characterised all states with principal quantum number, n, up to 6 and many states with $\mathrm{n} \leq 10$. Figure 1 shows the $\mathrm{N}_{2}^{+}\left(\mathrm{X}^{2} \Sigma_{\mathrm{g}}^{+}\right)$ground state which gives the the electronically bound limit of the system. For ${ }^{1} \Sigma_{\mathrm{u}}^{+}$symmetry, Rydberg series of the form $\mathrm{np} \sigma_{\mathrm{u}}$ and $\mathrm{nf} \sigma_{\mathrm{u}}$ converge to this limit. Comparison of the scattering and quantum chemistry-style curves shows good agreement for the lowest curve which shows a Rydberg-valence crossing at about $1.24 \AA$. However as only Rydberg states with low principle quantum number $(\mathrm{n} \leq 3)$ appear in the quantum chemistry calculation, the results start to diverge at the crossing around $-108.66 \mathrm{E}_{\mathrm{H}}$. This crossing is the result of the interaction between the $n=4$ Rydberg state and the valence state. In practice, one has to work very hard to use quantum chemistry packages such as MOLPRO to characterise even energetically low-lying regions containing Rydberg-valence crossings as attested by the very careful but limited study of Spelsberg and Meyer [30] and the more complete but less accurate studies of Hochlaf et al. [31, 32].

Figure 2 shows super excited states of nitrogen as a function of internuclear separation for ${ }^{1} \pi_{\mathrm{u}}$ symmetry. The states have been coloured according to their approximate partial wave character. Two distinct series are considered: the $\mathrm{np} \pi_{\mathrm{u}}$ and $\mathrm{nf} \pi_{\mathrm{u}}$ which converge on the $\mathrm{N}_{2}^{+}\left(\mathrm{X}^{2} \Sigma_{\mathrm{g}}^{+}\right)$state and the $\mathrm{ns} \sigma_{\mathrm{g}}$, $\mathrm{nd} \sigma_{\mathrm{g}}, \mathrm{ng} \sigma_{\mathrm{g}}, \mathrm{nd} \delta_{\mathrm{g}}$ and $\mathrm{ng} \delta_{\mathrm{g}}$ which converge $\mathrm{N}_{2}^{+}\left(\mathrm{B}^{2} \Pi_{\mathrm{u}}\right)$. The higher $\ell$ states have quantum defects which are near zero; this means that in figure 2 the $\mathrm{ng} \sigma_{\mathrm{g}}$ and $\mathrm{ng} \delta_{\mathrm{g}}$ states lie too close together to be separated, although both are given by the calculation.

As shown in Fig. 2, the character of the states changes at avoided crossings. Our calculations resolve avoided crossings not only between the purely bound states but also between resonances: see the region 


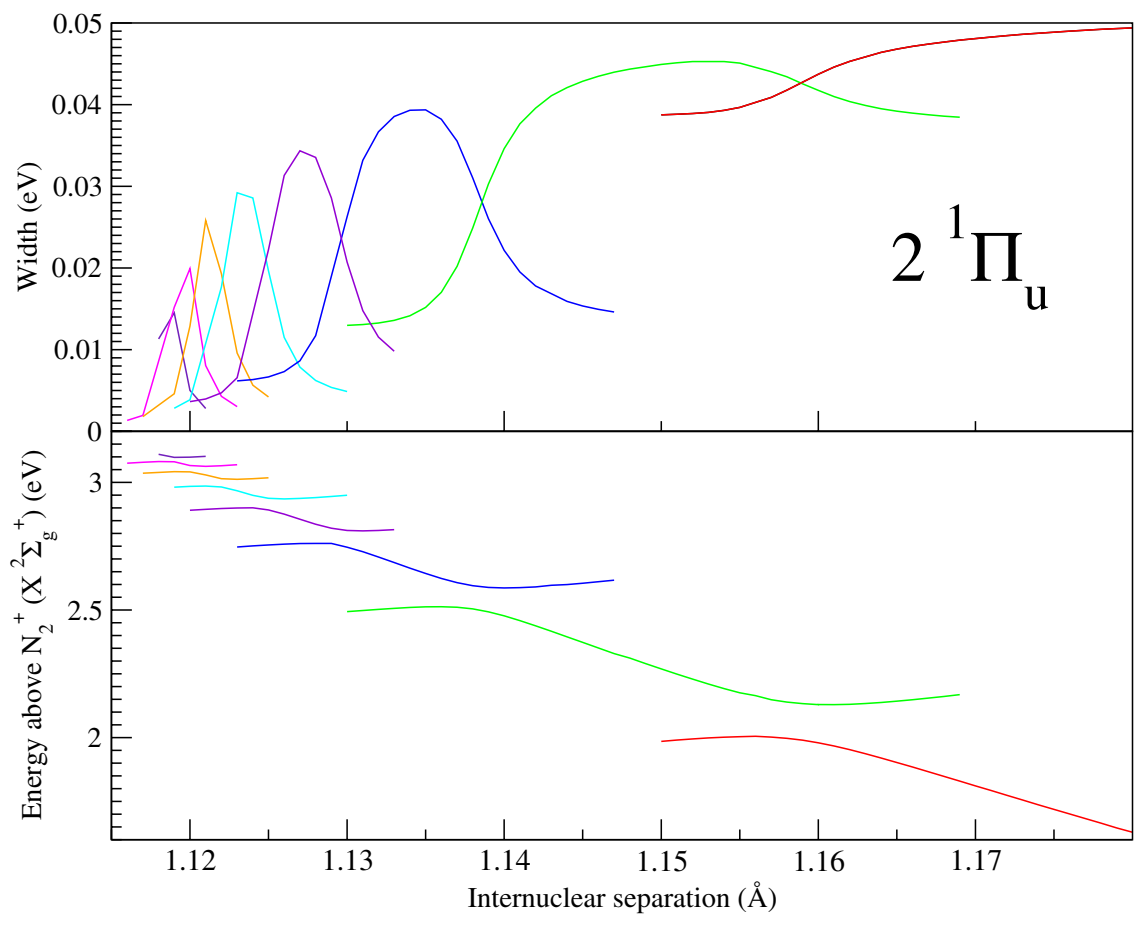

Figure 3. Resonance position (lower panel) and width (upper panel) for $\mathrm{N}_{2}$ with ${ }^{1} \Pi_{\mathrm{u}}$ symmetry. The figure shows the main dissociative curve as it passes through the ${ }^{1} \Pi_{\mathrm{u}}$-symmetry Rydberg series associated with the $\mathrm{N}_{2}^{+} A^{2} \Sigma_{\mathrm{u}}^{+}$ state.

between the two ion curves in Fig. 2 and the detailed depiction in Fig. 3. Such avoided crossings, which have largely been ignored in treatments of DR, also lead to pronounced structures in the resonance widths [10]. What is clear from the figure is that the "classic" dissociating curves which are associated with the direct mechanism for DR undergo multiple crossings, indeed an infinite number of them although only the first few are resolved, in both the bound and continuum regions.

\section{Discussion}

The results presented above represent a self-consistent model for calculating the four components generally considered as being essential for a DR calculation; the ground state ion potential energy curve (and excited state curves for core-excited), the dissociative neutral curves (bound and in the continuum) with asymptotic limits, the quantum defects of the Rydberg series converging on ionic states and, the couplings obtained through the resonant widths and diabatization of avoided crossings of bound states. This is as opposed to the previous calculation using MRCI curves by Guberman; the ion ground state is not explicitly included in the calculation and placed at an experimentally obtained energy, not an energy that is determined by the accuracy of the calculation. We hope to produce a truly ab initio rate for the DR of $\mathrm{N}_{2}^{+}$without empirical intervention.

In terms of positions and energies of potential energy curves, generally the agreement with Guberman is good (see [10] for an extensive comparison); however, some of our calculated widths are only about half as large [10] for the main dissociative curves of ${ }^{3} \Pi_{u}$ symmetry. Due to the many competing effects important to DR it is difficult to say conclusively what affect these lower widths will have on the rate, but it seems reasonable to say it will be slower. 
Core-excited Rydberg states play an important role in the DR of $\mathrm{N}_{2}^{+}$due to the proximity of the first excited state to the ground state as shown by Guberman [6]. The numerical implementation of multichannel quantum defect theory (MQDT) we plan to use, as used in a previous successful study of NO [20], currently does not take the core-excited states into account; this must be remedied if a rate to which Guberman's can be compared is to be found.

\section{Conclusion}

We have computed bound and continuum curves for super excited states of the nitrogen molecule. These curves will form the input for a MQDT treatment of dissociative recombination (DR) [33] for this key system. This work will follow a similar study for NO in which R-matrix data [20] was used as input for MQDT calculations. This study gave excellent agreement with experiment [34] and was then extended to treat processes involving vibrationally excited target states [35]. The calculations on NO however, relied on adjusting the position of the ab initio curves after comparison with spectroscopic data for the NO Rydberg states. The hope is that the model we have developed for superexcited states of $\mathrm{N}_{2}$ will be accurate enough that this recalibration step will not be required.

We thank Themisys Limited for supporting a studentship for DAL.

\section{References}

[1] A. Bultel, J. Annaloro, Plasma Sources Science and Technology 22, 025008 (2013)

[2] J.R. Peterson, A. Le Padellec, H. Danared, G.H. Dunn, M. Larsson, A. Larson, R.Peverall, C. Stromholm, S. Rosen, M. af Ugglas et al., J. Chem. Phys. 108, 1978 (1998)

[3] M. Larsson, A.E. Orel, Dissociative Recombination of Molecular Ions, Cambridge Molecular Science (Cambridge University Press, 2008), ISBN 9780521828192

[4] C.H. Sheehan, J.P. St.-Maurice, J. Geophys. Res.: Space Phys. 109, A03302 (2004)

[5] S. Guberman, The dissociative recombination of $\mathrm{N}_{2}^{+}$, in Dissocative Recombination of Molecular Ions with Electrons (Kluwer/Plenum Publishers, New York, 2003), pp. 187-196

[6] S.L. Guberman, J. Phys. Chem. A 111, 11254 (2007)

[7] S.L. Guberman, J. Chem. Phys. 137, 074309 (2012)

[8] S.L. Guberman, J. Chem. Phys. 139, 124318 (2013)

[9] H.J. Werner, P.J. Knowles, R. Lindh, F.R. Manby, M. Schütz et al., MOLPRO, a package of ab initio programs (2010), see http: //www . molpro.net/

[10] D.A. Little, J. Tennyson, J. Phys. B: At. Mol. Opt. Phys. 46, 145102 (2013)

[11] A. Faure, J.D. Gorfinkiel, L.A. Morgan, J. Tennyson, Comput. Phys. Commun. 144, 224 (2002)

[12] J. Tennyson, Comput. Phys. Commun. 100, 26 (1997)

[13] B.K. Sarpal, J. Tennyson, J. Phys. B: At. Mol. Opt. Phys. 25, L49 (1992)

[14] J. Tennyson, Phys. Rep. 491, 29 (2010)

[15] J.M. Carr, P.G. Galiatsatos, J.D. Gorfinkiel, A.G. Harvey, M.A. Lysaght, D. Madden, Z. Masin, M. Plummer, J. Tennyson, Eur. Phys. J. D 66, 58 (2012)

[16] J. Tennyson, J. Phys. B: At. Mol. Opt. Phys. 29, 1817 (1996)

[17] J. Tennyson, J. Phys. B: At. Mol. Opt. Phys. 37, 1061 (2004)

[18] R. Zhang, P. Galiatsatos, J. Tennyson, J. Phys. B: At. Mol. Opt. Phys. 44, 195203 (2011)

[19] B.K. Sarpal, S.E. Branchett, J. Tennyson, L.A. Morgan, J. Phys. B: At. Mol. Opt. Phys. 24, 3685 (1991)

[20] I. Rabadán, J. Tennyson, J. Phys. B: At. Mol. Opt. Phys. 30, 1975 (1997), erratum 31, 4485-4487 (1998) 
[21] D.T. Stibbe, J. Tennyson, Chem. Phys. Lett. 308, 532 (1999)

[22] J. Tennyson, C.J. Noble, Comput. Phys. Commun. 33, 421 (1984)

[23] A.U. Hazi, Phys. Rev. A 19, 920 (1979)

[24] D.T. Stibbe, J. Tennyson, Comput. Phys. Commun. 114, 236 (1998)

[25] F.T. Smith, Phys. Rev. 118, 349 (1960)

[26] D.T. Stibbe, J. Tennyson, J. Phys. B: At. Mol. Opt. Phys. 29, 4267 (1996)

[27] L. Quigley, K. Berrington, J. Pelan, Computer Physics Communications 114, 225 (1998)

[28] L. Quigley, K. Berrington, J. Phys. B: At. Mol. Opt. Phys. 29, 4529 (1996)

[29] I. Rabadán, J. Tennyson, J. Phys. B: At. Mol. Opt. Phys. 29, 3747 (1996)

[30] D. Spelsberg, W. Meyer, J. Chem. Phys. 115, 6438 (2001)

[31] M. Hochlaf, H. Ndome, D. Hammoutène, M. Vervloet, J. Phys. B: At. Mol. Opt. Phys. 43, 245101 (2010)

[32] M. Hochlaf, H. Ndome, D. Hammoutène, J. Chem. Phys. 132, 104310 (2010)

[33] V. Ngassam, A. Florescu, L. Pichl, I.F. Schneider, O. Motapon, A. Suzor-Weiner, Eur. Phys. J. D 26, 165 (2003)

[34] I.F. Schneider, I. Rabadán, L. Carata, J. Tennyson, L.H. Andersen, A. Suzor-Weiner, J. Phys. B: At. Mol. Opt. Phys. 33, 4849 (2000)

[35] O. Motapon, M. Fifirig, A. Florescu, F.O. Waffeu-Tamo, O. Crumeyrolle, G. Varin-Breant, A. Bultel, P. Vervisch, J. Tennyson, I.F. Schneider, Plasma Sources Sci. Technol. 15, 23 (2006) 